\title{
TUMORES DEL ESTROMA GASTROINTESTINAL (GIST) DEL DUODENO: PRESENTACIÓN CLÍNICA, ESTUDIO DIAGNÓSTICO Y TRATAMIENTO ACTUAL*
}

\author{
Dr. Marcelo A. Beltrán S. ${ }^{1,2}$ \\ 1 Servicio de Cirugía, Hospital de La Serena. \\ 2 Facultad de Medicina, Departamento de Clínicas, Universidad Católica del Norte, Coquimbo, Chile. \\ Chile.
}

\begin{abstract}
Duodenal gastrointestinal stromal tumors (GIST): Clinical presentation, diagnostic studies and current treatment

Duodenal gastrointestinal stromal tumors (GIST) constitute the most challenging location for the treatment of this neoplasm. Duodenal GIST are relatively uncommon tumors, their prevalence is very low accounting for $5 \%$ to $7 \%$ or less of all surgically resected GIST. Most published reports on duodenal GIST are case reports or case series. Consequently, the clinical manifestations, radiologic diagnosis, appropriate surgical treatment, and prognostic factors constitute a subject of current controversy. Most articles concerning duodenal GISTs state that unlike tumors involving other sites of the gastrointestinal tract, the optimal procedure for duodenal GISTs has not been well characterized. However, when carefully reviewing the published literature on the subject, it was found that surgical approaches to duodenal GISTs are fairly standard among different authors. All take into account the location of GIST in the duodenum and its anatomic relationships to decide whether local resection or Whipple operation should be performed. Using this common sense knowledge, defined surgical options for duodenal GISTs according to their localization within the duodenal frame are proposed.
\end{abstract}

Key words: Duodenum, gastrointestinal stromal tumors, duodenal GIST, duodenal tumors.

\section{Resumen}

Los tumores del estroma gastrointestinal (GIST) localizados en el duodeno, constituyen la localización más compleja para el tratamiento de esta neoplasia. Los GIST duodenales son relativamente infrecuentes con una prevalencia de 5\% a 7\% de todos los GIST tratados quirúrgicamente. La mayoría de los reportes publicados sobre GIST duodenal son reportes de caso o series de casos. Consecuentemente, las manifestaciones clínicas, el diagnóstico radiológico, el tratamiento quirúrgico y los factores pronósticos constituyen materia de

\footnotetext{
Los autores no refieren conflictos de interés

Correspondencia: Dr. Marcelo A. Beltrán S. beltran_01@yahoo.com
}

*Recibido el 4 de noviembre de 2013 y aceptado para publicación el 2 de diciembre de 2013. 
controversia. La mayoría de los artículos sobre GIST duodenales mencionan que a diferencia de otros tumores localizados en el tracto gastrointestinal, el procedimiento óptimo para el tratamiento del GIST duodenal no se encuentra bien caracterizado en la literatura. Sin embargo, la revisión de las publicaciones sobre el tema demuestra que el abordaje quirúrgico descrito por diferentes autores es bastante estándar. Todos toman en cuenta la localización del GIST en el duodeno y sus relaciones anatómicas para decidir entre la resección local o la pancreatoduodenectomía. Utilizando este conocimiento de sentido común, se proponen opciones quirúrgicas definidas para GIST duodenales basadas en su localización en el duodeno.

Palabras clave: Duodeno, tumores del estroma gastrointestinal, GIST duodenal, tumores duodenales.

\section{Introducción}

Los tumores del estroma gastrointestinal (GIST) se definen como tumores mesenquimatosos de células fusiformes, epiteloides y ocasionalmente pleomórficas, que se originan en el tracto gastrointestinal, expresan la proteína KIT (c-kit), reaccionan positivamente a la tinción inmunohistoquímica con CD117 y son portadores de una mutación en un gen que codifica el receptor tipo III de tirosino-kinasa ${ }^{1}$. Los GIST se conocen como tales desde $1983^{2}$. En 1998 se definió el rol del c-kit en la patología del GIST $^{3}$. El año 2001, Joensuu publicó un artículo seminal sobre la actividad del mesilato de imatinib sobre los GIST ${ }^{4}$. El manejo actual incluye la cirugía para tumores localizados y la combinación de imatinib y cirugía para tumores recurrentes o metastásicos. Los estudios de imagen recomendados son la tomografía computarizada (TC), la resonancia magnética $(\mathrm{RM})$ y la tomografía por emisión de positrones (PET) $)^{5,6}$. Actualmente, el estudio radiológico de elección en la evaluación de los pacientes con sospecha de GIST es la TC ${ }^{5}$. La incidencia general de los GIST varía entre 10 a 20 casos por millón de personas por año $0^{5-11}$. Constituyen el tumor mesenquimatoso más común del sistema gastrointestinal y cuentan por $1 \%$ a $2 \%$ de todas las neoplasias gastrointestinales ${ }^{1-7}$. Se desarrollan a lo largo de todo el tracto gastrointestinal; son más frecuentes en el estómago ( $50 \%$ a $70 \%)$, seguidos del yeyuno e íleon $(20 \%$ a $30 \%)$, colon, recto y apéndice $(0,5 \%$ a $10 \%)$ y esófago $(1 \%)^{1-10}$. Se han descrito casos de GIST extra-gastrointestinales localizados en el páncreas, vesícula biliar, mesenterio, omento mayor y menor y retroperitoneo $(1,5 \% \text { a } 5 \%)^{7,8}$. Los GIST duodenales son relativamente infrecuentes (prevalencia 5\% a 7\%), sin embargo, representan 30\% de todos los tumores duodenales primarios ${ }^{7}$. La mayoría de las publicaciones sobre GIST duodenales son reportes de casos o series de $\operatorname{casos}^{7-21}$. Consecuentemente, las manifestaciones clínicas, estudio y tratamiento constituyen materia de controversia. El presente artículo revisa el tratamiento quirúrgico de los GIST duodenales tratando de establecer opciones quirúrgicas de acuerdo a la localización del tumor en el duodeno.

\section{Biología}

Los GIST se originan en las células intersticiales de Cajal que forman una malla celular alrededor del plexo mientérico y muscular propia de la pared gastrointestinal ${ }^{1-8}$. La mayoría se componen de células fusiformes $(70 \%)$, otros presentan predominantemente células epiteloides $(20 \%)$ y el restante $10 \%$ consiste en una mezcla de células fusiformes y epiteloides ${ }^{8,9}$. La morfología celular varía según el sitio de origen y la mutación de los genes del KIT y del factor de crecimiento plaquetario alfa $(\mathrm{PDGFR} \alpha)^{10-12}$. En $10 \%$ no se encuentran mutaciones del $\mathrm{KIT}^{10}$. Aproximadamente $86 \%$ a $95 \%$ reaccionan al CD117; entre $70 \%$ y $81 \%$ a CD34; de $35 \%$ a $70 \%$ a la actina de músculo liso; entre $10 \%$ y $38 \%$ a S- 100 y $5 \%$ a desmina ${ }^{1-9}$. Un gen recientemente caracterizado que codifica la proteína DOG1, reacciona positivamente al anticuerpo anti-DOG1 y permite identificar GIST con mutaciones del KIT o del PDGFR- $\alpha^{10}$. En $95 \%$ es posible encontrar mutaciones del KIT o del PDGFR $\alpha^{10,11}$. El KIT codificado por el gen $c$-kit es una glicoproteína de la membrana celular de la subclase III de la familia de receptores de las tirosino-kinasa, estructuralmente similar al PDGFR $\alpha^{9,10}$. La función del KIT es crítica para el desarrollo de las células intersticiales de Cajal, células progenitoras hematopoyéticas, mastocitos y células germinativas. La mutación del KIT es un evento temprano en el desarrollo de los GIST, siendo la mutación del exón 11 la más común $(92 \%)^{10}$. Las mutaciones del exón 9 son más frecuentes en el intestino delgado (95\%) y se encuentran en $10 \%$ a $18 \%$ de todos los GIST $^{9}$. La mayoría de los GIST duodenales tienen mutaciones del exón 11 (40\% a $70 \%$ ), otras mutaciones están localizadas en el exón $9(13 \%$ a $31 \%)$ y en el exón $13(11 \%)$; en $10 \%$ a $15 \%$ no se encuentran mutaciones. Las mutaciones del KIT tienen implicaciones en el pronóstico; las mutaciones del exón 11 se encuentran frecuentemente en GIST con elevado riesgo de malignidad, las mutaciones del exón 9 se presentan en GIST malignos $^{9}$ (Tabla 1). 
Tabla 1. Características moleculares y clasificación genética ${ }^{9, *}$

\begin{tabular}{|ll|}
\hline Tipo de GIST & Características \\
Esporádico & \\
Mutación KIT & \\
Exón 11 & La mejor respuesta a imatinib \\
Exón 9 & Respuesta intermedia a imatinib \\
Exón 13 & Sensible in vitro, repuestas clínicas observadas \\
Exón 17 & Sensible in vitro, respuestas clínicas observadas \\
Mutación PDGFR- $\boldsymbol{a}$ & \\
Exón 12 & Sensible in vitro, respuestas clínicas observadas \\
Exón 18 & D842V mala respuesta, otras mutaciones son sensibles \\
Salvaje & \\
GIST Familiar & \\
KIT exón 11 & Pigmentación de la piel, urticaria, mastocitosis \\
KIT exón 13 & Sin pigmentación de la piel o mastocitosis \\
KIT exón 17 & Sin pigmentación de la piel o mastocitosis \\
GIST con paraganglioma & Frecuentemente síntomas endocrinos \\
GIST Pediátrico & \\
Esporádico & Infrecuentes mutaciones del KIT \\
Triada de Carney & GIST gástrico, condroma pulmonar y paraganglioma. No se han identificado mutaciones del KIT \\
\hline GIST asociado a NF-1 & No se han identificado mutaciones del KIT \\
\hline
\end{tabular}

NF-1: Neurofibromatosis tipo 1. *Según Corless CL, et al.

\section{Presentación clínica}

Los GIST se manifiestan clínicamente alrededor de los 60 años de edad, siendo más frecuentes en pacientes masculinos $(68 \% \text { a } 80 \%)^{7-21}$. La localización duodenal no es común con una prevalencia de $5 \%$. $\%^{78}$. En series de casos de GIST localizados específicamente en el duodeno la incidencia varía entre $6 \%$ y $29 \%{ }^{16,17,19}$. Debido a la ausencia de manifestaciones clínicas tempranas, los GIST duodenales se presentan cuando han alcanzado un tamaño que produce síntomas relacionados al efecto de masa o hemorragia digestiva ${ }^{13-21}$. Con mayor frecuencia son asintomáticos y son descubiertos incidentalmente durante estudios endoscópicos o radiológicos por otras causas ${ }^{13-23}$. Los GIST duodenales se originan con mayor frecuencia en la segunda porción del duodeno $(33 \%$ a $64 \%)$, seguidos en orden por la tercera $(22 \%$ a $42 \%)$, cuarta $(8 \%$ a $25 \%)$ y primera $(5 \%$ a $21 \%$ ) porciones ${ }^{7-20}$. Los síntomas más comunes son la hemorragia digestiva y el dolor abdominal ${ }^{17-20}$. Aun así muchos GIST son asintomáticos (Tabla 2). La mayoría se presentan con hemorragia digestiva alta macroscópica u oculta ${ }^{19-21}$. La hemorragia se debe a ulceración de tumores de crecimiento endoluminal ${ }^{18,19}$. El dolor abdominal se presenta en GIST grandes de crecimiento exofítico y se debe al efecto de masa y a la compresión de los órganos adyacentes $^{19}$. De acuerdo a los criterios de riesgo
Tabla 2. Presentación clínica de los GIST duodenales ${ }^{7-20,42-45}$

\begin{tabular}{|lc|}
\hline Características & Frecuencia (\%) \\
\hline Asintomático/hallazgo incidental & $9-33$ \\
Hemorragia/anemia & $22-100$ \\
Dolor abdominal & $16-45$ \\
Masa abdominal palpable & $4-18$ \\
Pérdida de peso & $2-14$ \\
Ictericia & $9-11$ \\
Anorexia & $1-9$ \\
Obstrucción intestinal & $1-3$ \\
\hline
\end{tabular}

de progresión para GIST ${ }^{22,23}$ (Tabla 3), los GIST duodenales presentan muy bajo riesgo en $8 \%$ de los casos, bajo riesgo en 31\% y alto riesgo en $69 \%$ de los $\operatorname{casos}^{21,23}$. En el momento de su presentación clínica, la mayoría de los GIST duodenales (89\%) son tumores solitarios ${ }^{19}$.

\section{GIST duodenales en pacientes con neurofibromatosis tipo I}

La mayoría de los GIST son esporádicos. En $5 \%$ de los casos se presentan en el contexto de un 
Tabla 3. Pronóstico de GIST primarios modificada por Joensuu ${ }^{22,23}$

\begin{tabular}{|llcl|}
\hline Riesgo & Tamaño $(\mathbf{c m})$ & Recuento de las mitosis (por 50 CAM*) & Localización del tumor \\
Muy bajo riesgo & $<2$ & $<5$ & Cualquiera \\
Bajo riesgo & $2-5$ & $<5$ & Cualquiera \\
Riesgo intermediario & $<5$ & $6-10$ & Gástrico \\
& $5-10$ & $<5$ & Gástrico \\
Alto riesgo & Cualquiera & Cualquiera & Ruptura del tumor \\
& $>10$ & Cualquiera & Cualquiera \\
& Cualquiera & $>10$ & Cualquiera \\
& $>5$ & $>5$ & Cualquiera \\
& $>5$ & $>5$ & No gástrico \\
& $5-10$ & $>5$ & No gástrico \\
\hline
\end{tabular}

*CAM: campos de aumento mayor.

síndrome familiar siendo la neurofibromatosis tipo 1 (NF1) el más común ${ }^{21}$, además se describen en la triada de Carney y en el síndrome de Carney-Stra-

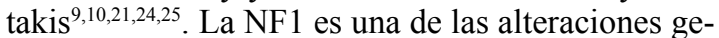
néticas más comunes con una frecuencia de 1:2.500 a 1:5.000 nacimientos ${ }^{24-27}$. Se hereda en un patrón autosómico dominante con penetración variable, la anomalía genética se localiza en el brazo largo del cromosoma 17 (17q11.2), este gen codifica la neurofibromina que es una proteína citoplásmica que controla la proliferación celular inactivando las vías del ras p21 y de la kinasa MAP ${ }^{26}$. El tipo de GIST que se desarrolla en NF1 resulta de la inactivación genética de la neurofibromina lo que resulta en la hiperactivación de la cascada del ras 21 y de la kinasa $\mathrm{MAP}^{10}$. En el GIST familiar se observa hiperplasia notoria de las células intersticiales de Cajal en el intestino delgado y colon. Normalmente, las células de Cajal comprenden una o dos capas celulares, en los pacientes con GIST se observan 10 ó 20 capas celulares ${ }^{10}$. Las complicaciones gastrointestinales de la NF1 se encuentran extensamente descritas pero la verdadera incidencia y repercusión clínica de los GIST no son conocidas ${ }^{27}$. Se ha estimado que una proporción que varía entre $10 \%$ y $60 \%$ de todos los pacientes con NF1 desarrollan GIST, aunque sólo $5 \%$ o menos presentan sintomatología asociada ${ }^{24}$. Los GIST duodenales son infrecuentes en pacientes con NF1 ${ }^{24}$. En estos pacientes los GIST se localizan en el intestino delgado, con mayor frecuencia en el íleon ${ }^{24-27}$, son habitualmente múltiples coexistiendo tumores benignos y malignos ${ }^{23}$.

\section{Diagnóstico}

\section{Endoscopía}

La endoscopía convencional visualiza la primera y segunda porciones del duodeno. Los GIST se observan como tumores submucosos elevados bien delimitados y ocasionalmente ulcerados ${ }^{7,17,18,21}$. Son fácilmente identificados en la primera y segunda porciones duodenales. Ocasionalmente pequeños GIST ulcerados menores a $2 \mathrm{~cm}$ han sido confundidos con la papila de Vater sospechando hemobilia ${ }^{18}$. La biopsia endoscópica raramente permite el diagnóstico correcto mostrando inflamación crónica o mucosa duodenal normal. Se ha reportado hasta $21 \%$ de diagnóstico correcto con biopsias duodenales profundas?

\section{Tomografía computarizada}

El estudio radiológico con mayor disponibilidad en la actualidad para identificar y diagnosticar GIST es la tomografía computarizada (CT). Prácticamente todos los GIST duodenales son detectados con $\mathrm{CT}^{17,18}$. Los GIST son tumores hipervasculares que en imágenes contrastadas se contrastan intensamente en la fase arterial ${ }^{15}$. La mayoría se contrastan homogéneamente y aparecen como masas endofíticas o exofíticas bien definidas ${ }^{28-31}$. Los tumores pequeños se muestran como masas homogéneas bien delineadas ovaladas o redondas moderadamente contrastadas $^{28,29,32-34}$ (Figura 1A). Los GIST grandes pueden presentar ulceración de la mucosa, se observan bien contrastados, bien delimitados, lobulados y heterogéneos, presentan cavitación y líquido por necrosis central y crecimiento endoluminal o extraluminal ${ }^{7,17,28,29,31,32}$ (Figura 2). Los criterios tomográficos para la descripción y registro de GIST fueron definidos por Burkill y cols ${ }^{29,30}$. Los GIST evidentemente malignos se presentan con metástasis hepáticas o con invasión local de los tejidos y órganos circundantes ${ }^{28-35}$. El diagnóstico diferencial incluye otros tumores de tejidos blandos como leiomioma, leiomiosarcoma, mesenteritis fibrosa, linfangioma mesentérico, neurofibromatosis plexiforme, páncreas 
ectópico y tumores malignos de la vaina nerviosa y de origen vascular ${ }^{29-31}$.

\section{Resonancia magnética}

Las imágenes de la resonancia magnética (RM) son comparables a las de la $\mathrm{CT}^{17-30}$. En RM los GIST duodenales sólidos tienen una señal de intensidad baja en $\mathrm{T} 1$ y elevada en $\mathrm{T} 2^{7,15,33}$. La necrosis y la hemorragia dentro del tumor influencian la intensidad de las imágenes de la $\mathrm{RM}^{17}$.

\section{Endosonografia}

Este estudio no se encuentra ampliamente disponible. Puede ser utilizado para identificar la localización exacta del tumor dentro de la pared duodenal y sus relaciones con estructuras adyacentes, principalmente con la papila de Vater y la cabeza del páncreas.

\section{Terapia neoadyuvante}

El primer inhibidor exitoso de moléculas pequeñas fue el mesilato de imatinib, desarrollado como inhibidor específico de PDGFR $\alpha^{36}$. Posteriormente se descubrió que actuaba como un potente inhibidor del KIT salvaje y varios tipos de KIT mutado que se encuentran en los GIST ${ }^{10,36,37}$. Los GIST se desarrollan con frecuencia en adultos con mutaciones de la línea germinal del KIT, la mayoría de los pacientes que no tienen mutaciones del KIT tienen mutaciones de PDGFR $\alpha^{36}$. Los GIST con mutaciones del exón 11 tienen la mejor respuesta al imatinib, los pacientes sin mutaciones del KIT o de PDGFR $\alpha$ no responden al imatinib ${ }^{36,37}$. El primer reporte del uso exitoso de imatinib en el tratamiento de un paciente con GIST avanzado fue publicado el año $2001^{4}$, inmediatamente después se desarrollaron estudios clí-
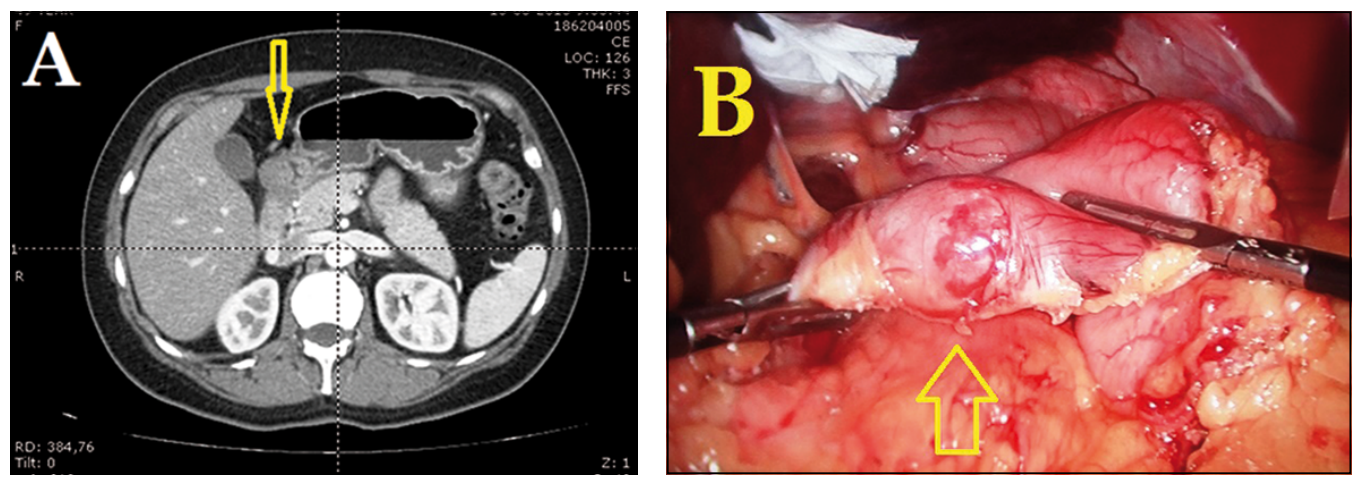

Figura 1. A. GIST de la primera porción del duodeno homogéneamente contrastado que se presentó en una mujer de 45 años de edad. El tumor se muestra como una masa ovalada, bien delineada, endofítica, homogénea y moderadamente contrastada. El tumor medía $21 \mathrm{~mm}$ de diámetro, la paciente fue operada mediante gastrectomía distal laparoscópica que incluyó la primera porción del duodeno. B. Visión laparoscópica del antro gástrico y primera porción del duodeno donde se observa claramente la tumoración del GIST.
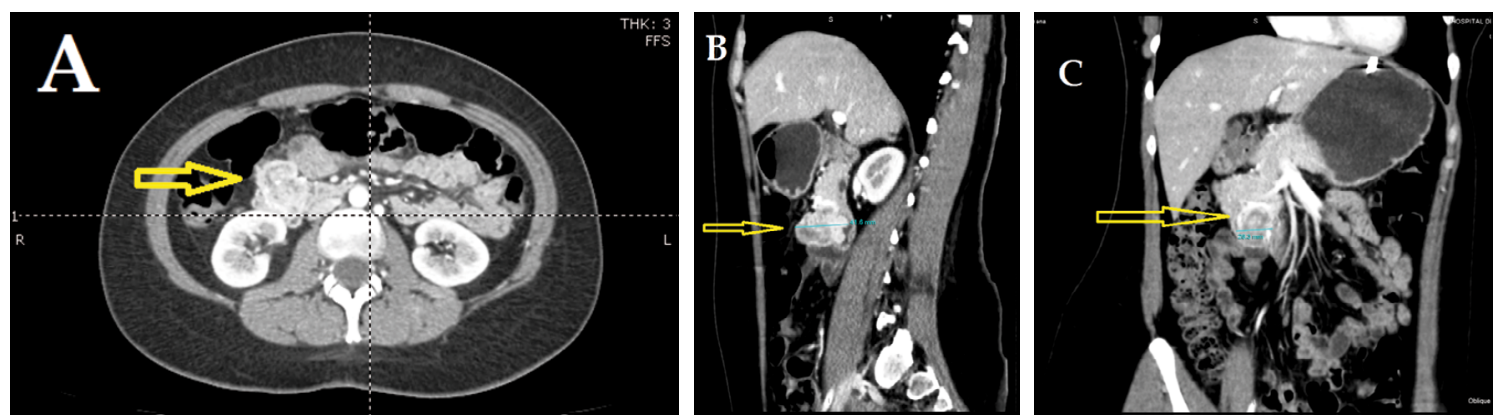

Figura 2. A. GIST duodenal de la segunda porción heterogéneamente contrastado en la fase arterial, que se presentó en una paciente de 40 años de edad. En la vista transversal se muestra el tumor bien definido en la pared medial de la segunda porción duodenal infiltrando la cabeza del páncreas. La paciente fue tratada mediante pancreatoduodenectomía. B. Vista lateral que muestra el GIST duodenal que mide $4 \mathrm{~cm}$ en su dimensión lateral. C. Vista coronal que muestra el GIST duodenal que mide $3 \mathrm{~cm}$ en su dimensión frontal. 
nicos ${ }^{37}$, los que llevaron a la aprobación del imatinib por la United States Food and Drug Administration para tratamiento de GIST el año $2002^{10}$. Las indicaciones de terapia neoadyuvante con imatinib son la reducción tumoral preoperatoria ${ }^{10,38}$ y el tratamiento de tumores metastásicos avanzados ${ }^{37}$. En casos seleccionados de GIST localmente avanzado o marginalmente resectable, la estrategia de citoreducción con imatinib neoadyuvante se ha establecido como una práctica común ${ }^{39-41}$. El uso de imatinib neoadyuvante en la práctica rutinaria asociada a cirugía tiene excelentes resultados a largo plazo como ha sido demostrado en el estudio más importante de pacientes con GIST, en el cual 10\% tenían GIST duodenales ${ }^{39}$.

\section{Indicaciones para tratamiento neoadyuvante con imatinib}

La resección completa de grandes GIST es algunas veces difícil debido a razones técnicas. En estos casos, la terapia neoadyuvante con imatinib está indicada ${ }^{19,38,39}$. Los candidatos para imatinib preoperatorio son aquellos pacientes quienes se beneficiarían de la reducción del volumen tumoral antes de la operación; esta estrategia es especialmente atractiva en tumores con localizaciones complejas como los GIST duodenales de la segunda y tercera porción ${ }^{39,40}$. Después de la reducción tumoral, será posible la resección exitosa hasta en $80 \%$ de los $\operatorname{casos}^{38,39}$. El imatinib ha sido utilizado en el preoperatorio con buenos resultados en pacientes con GIST duodenales grandes candidatos a pancreatoduodenectomía ${ }^{19,20,39,41}$. En estos casos además de la reducción tumoral el imatinib reduce la fragilidad del GIST disminuyendo el riesgo de hemorragia y la posibilidad de rompimiento de la cápsula ${ }^{20,39}$.

\section{Dosis y manejo de la terapia neoadyuvante con imatinib}

La dosis recomendada de imatinib es de 400 mg diarios hasta alcanzar la respuesta máxima que se confirma con dos TC consecutivas que no muestren mayor regresión del tumor o hasta que el cirujano defina como posible una resección económica, cualquiera de ambas situaciones sea primero alcanzada $^{38,39}$. La duración óptima de la terapia preoperatoria varía entre 4 y 12 meses para alcanzar la respuesta máxima.

\section{Cirugía}

La resección quirúrgica completa constituye el único tratamiento curativo para los GIST ${ }^{10,17,39,42-45}$. El tratamiento quirúrgico óptimo incluye la resección del tumor con márgenes libres que puede incluir los órganos adyacentes ${ }^{44}$. Los GIST no se di- seminan por vía linfática y no presentan crecimiento submucoso, además son tumores bien encapsulados que raramente invaden órganos vecinos, por lo que la resección local o la duodenectomía segmentaria se considera suficiente y se asocia a una prolongada supervivencia libre de enfermedad ${ }^{10-21,43,44,46}$. A diferencia de los GIST localizados en otros lugares del tracto gastrointestinal, el tratamiento quirúrgico óptimo para GIST duodenales no se encuentra bien caracterizado y está pobremente definido en la literatura quirúrgica ${ }^{17,19,21}$. La resección quirúrgica puede llevarse a cabo mediante varias opciones ${ }^{17,42,43,46}$. El abordaje debe estar dictado por la localización del tumor en el duodeno y por la capacidad para lograr una resección $\mathrm{R} 0^{19,43}$. La cirugía local o conservadora se define como la resección en cuña o la resección de un segmento duodenal y aun cuando sea técnicamente posible deben tenerse en cuenta la anatomía duodenal y su proximidad a estructuras cruciales como la papila de Vater, el páncreas, los vasos mesentéricos, el colédoco y el conducto pancreático $^{7,19,20,26,44,46}$. Consecuentemente, la resección local muchas veces es difícil, especialmente en la segunda porción ${ }^{16,19}$. En la práctica clínica, hasta $86 \%$ de los GIST duodenales han sido tratados mediante pancreatoduodenectomía ${ }^{44}$. Las opciones quirúrgicas reportadas para GIST duodenales dependen de la localización del tumor y de su tamaño ${ }^{15-21,24,25,42-54}$ (Tabla 4).

\section{Primera porción del duodeno}

Los tumores de la primera porción duodenal localizados hacia la pared medial que se encuentran en contacto íntimo con la cabeza del páncreas deben ser tratados mediante pancreatoduodenectomía ${ }^{16,19,20,25,26,42,43,45}$. Los GIST localizados en la pared lateral cuando son menores a $3 \mathrm{~cm}$ (Figura 1), pueden ser tratados mediante resección local en cuña y sutura primaria o resección segmentaria ${ }^{17,26,42,43,45,47}$ (Figura 3).

\section{Segunda porción del duodeno}

La decisión de realizar una pancreatoduodenectomía en pacientes con GIST de la segunda porción duodenal se encuentra influenciada por las relaciones del tumor con la cabeza del páncreas y con la papila de Vater. Los GIST duodenales localizados en la pared medial del duodeno en contacto con la cabeza del páncreas o con la papila de Vater deben ser sometidos a pancreatoduodenectomía ${ }^{16,19,20,25,26,38,42,48,50}$. Los tumores localizados en la pared lateral de la segunda porción pueden ser tratados mediante resección local en cuña con cierre primario o duodenectomía distal y reconstrucción mediante anastomosis entre el yeyuno y el duodeno restante o mediante anastomosis duodeno-yeyunal 
Tabla 4. Procedimientos quirúrgicos recomendados de acuerdo a la localización del GIST en el duodeno ${ }^{15-21,24,25,38,42-54}$

\begin{tabular}{|c|c|c|c|c|}
\hline Porción & Primera & Segunda & Tercera & Cuarta \\
\hline \multicolumn{5}{|l|}{ Pared lateral } \\
\hline GIST $<3 \mathrm{~cm}$ & $\begin{array}{l}\text { - Resección en cuña } \\
\text { - Resección segmentaria } \\
\text { + Billroth I/Gastro- } \\
\text { yeyunostomía en Y de } \\
\text { Roux }\end{array}$ & $\begin{array}{l}\text { - Resección en cuña + } \\
\text { cierre primario / Duo- } \\
\text { deno-yeyunostomía en } \\
\text { Y de Roux }\end{array}$ & $\begin{array}{l}\text { - Resección en cuña + } \\
\text { cierre primario / Duo- } \\
\text { deno-yeyunostomía en } \\
\text { Y de Roux }\end{array}$ & $\begin{array}{l}\text { - Resección en cuña } \\
\text { - Resección segmentaria } \\
\text { + duodeno-yeyunosto- } \\
\text { mía primaria }\end{array}$ \\
\hline GIST $>3 \mathrm{~cm}$ & $\begin{array}{l}\text { - Resección segmen- } \\
\text { taria/antrectomía + } \\
\text { gastro-yeyunostomía } \\
\text { en Y de Roux } \\
\text { - Whipple }\end{array}$ & - Whipple & $\begin{array}{l}\text { - Whipple } \\
\text { - Resección segmentaria } \\
\text { + duodeno-duodenos- } \\
\text { tomía } \\
\text { - Resección de la terce- } \\
\text { ra y cuarta porción + } \\
\text { duodeno-yeyunostomía }\end{array}$ & $\begin{array}{l}\text { - Resección segmentaria } \\
\text { + duodeno-yeyunosto- } \\
\text { mía primaria }\end{array}$ \\
\hline \multicolumn{5}{|l|}{ Pared medial } \\
\hline GIST $<3 \mathrm{~cm}$ & $\begin{array}{l}\text { - Resección en cuña } \\
\text { - Resección segmentaria } \\
\text { + Billroth I/Gastro- } \\
\text { yeyunostomía en Y de } \\
\text { Roux }\end{array}$ & - Whipple & $\begin{array}{l}\text { - Whipple } \\
\text { - Resección segmentaria } \\
\text { + duodeno-duodenos- } \\
\text { tomía } \\
\text { - Resección de la terce- } \\
\text { ra y cuarta porción + } \\
\text { duodeno-yeyunosto- } \\
\text { mía }\end{array}$ & $\begin{array}{l}\text { - Resección segmentaria } \\
\text { + duodeno-yeyunosto- } \\
\text { mía primaria }\end{array}$ \\
\hline GIST $>3 \mathrm{~cm}$ & - Whipple & - Whipple & - Whipple & $\begin{array}{l}\text { - Whipple } \\
\text { - Resección segmentaria } \\
\text { + duodeno-yeyunosto- } \\
\text { mía primaria }\end{array}$ \\
\hline
\end{tabular}
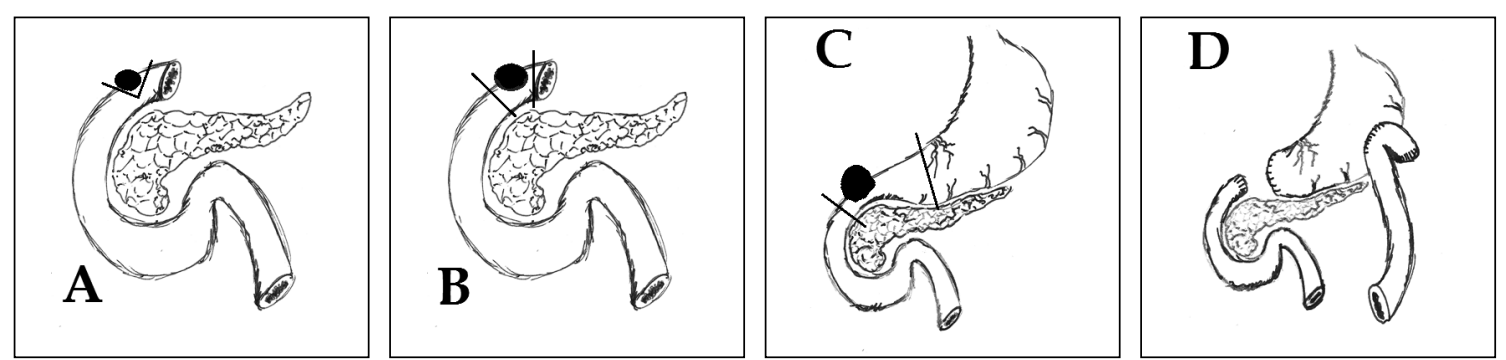

Figura 3. Procedimientos quirúrgicos para GIST de la primera porción duodenal A. Resección en cuña con sutura primaria. B. Resección segmentaria con anastomosis primaria. C. GIST que requiere resección segmentaria extensa. D. Reconstrucción con anastomosis gastro-yeyunal en Y de Roux después de resección segmentaria ampliada al antro gástrico.

en Y de Roux ${ }^{15,19,25,43,45,49}$ (Figura 4). La indicación de resección en cuña o segmentaria será dictada por el tamaño del tumor y por la posibilidad de lograr una resección con márgenes libres de tumor ${ }^{45}$. Cuando la patencia de la ampolla de Vater se encuentra en riesgo o cuando el margen de resección se encuentra muy cercano a esta estructura, se podría efectuar una papiloplastía para asegurar el flujo exocrino a través de la papila ${ }^{51}$.

\section{Tercera porción del duodeno}

Tumores pequeños o de mediano tamaño localizados en la pared medial o lateral de la tercera porción (Figura 5), podrían ser tratados mediante resección de la tercera y cuarta porción duodenal $\mathrm{y}$ anastomosis termino-terminal o latero-terminal primaria con el yeyuno o mediante resección local en cuña con cierre primario ${ }^{15,19,25,45,52,53}$ (Figuras 6A y 6B). Cuando la resección en cuña deja un defecto 
muy grande en la pared del duodeno y el cierre primario no es posible, el defecto puede resolverse mediante duodeno-yeyunostomía en $\mathrm{Y}$ de Roux ${ }^{45}$ (Figuras 6C, 6D, 6E, 6F, 6G y 6H). Cuando el tumor es muy grande y se encuentra en la pared medial en contacto o infiltrando el páncreas, el tratamiento mediante pancreatoduodenectomía es la mejor opción ${ }^{19}$.

\section{Cuarta porción del duodeno}

Los GIST localizados en la cuarta porción pueden ser resecados localmente y el tránsito intestinal restaurado mediante anastomosis primaria termino-terminal o latero-terminal entre la tercera porción duodenal y el yeyuno ${ }^{17,24,45}$ (Figura 7). Los tumores pequeños pueden ser tratados mediante resección en cuña ${ }^{54}$.

\section{Cirugía laparoscópica}

El abordaje laparoscópico se ha establecido indiscutiblemente para la mayoría de los GIST gástricos

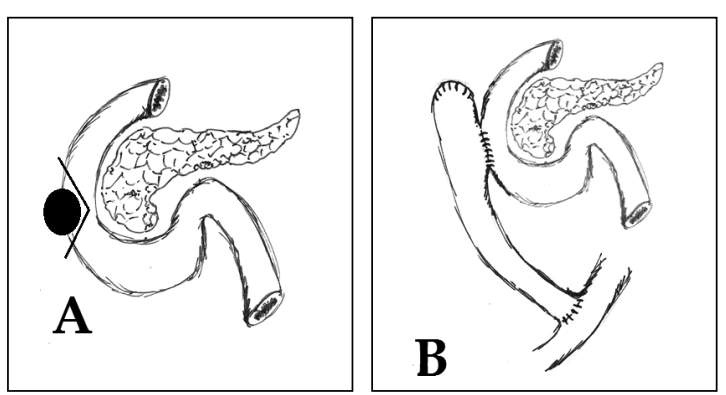

Figura 4. Procedimientos quirúrgicos para GIST de la segunda porción duodenal. A. Resección en cuña. B. Reconstrucción de una resección en cuña amplia de la segunda porción del duodeno mediante duodeno-yeyuno anastomosis. y está incluido dentro de los algoritmos formalmente propuestos para el tratamiento de estos tumores ${ }^{55}$. Respecto a los GIST duodenales la cirugía laparoscópica no se encuentra incluida en ningún algoritmo y sólo algunas experiencias anecdóticas han sido publicadas incluyendo dos GIST de la primera porción duodenal tratados mediante resección en cuña y cierre primario ${ }^{17}$. En la experiencia de nuestra institución en una ocasión se realizó resección laparoscópica de un GIST duodenal de la primera porción mediante antrectomía incluyendo la primera porción duodenal y el antro gástrico reconstruyendo el tránsito intestinal mediante gastro-yeyunostomía en Y de Roux (Figura 1). En GIST duodenales pequeños sin importar la porción duodenal donde se encuentren, se han reportado resecciones locales con excelentes resultados quirúrgicos y excelente sobrevivencia libre de enfermedad; consecuentemente, en tumores seleccionados el abordaje laparoscópico debe ser intentado. Tumores malignos y benignos de la cabeza del páncreas han sido exitosamente tratados mediante pancreatoduodenectomía laparoscópica; consecuentemente, tiene sentido intentar este abordaje en GIST de la segunda porción del duodeno con indicación de esta cirugía. Sin embargo, algunos autores sugieren excluir los GIST duodenales de los abordajes laparoscópicos ${ }^{56}$.

\section{Controversias quirúrgicas}

La decisión final de someter a un paciente a pancreatoduodenectomía por GIST duodenal depende no sólo de la localización del tumor sino también del tamaño $0^{20,44}$. Los GIST duodenales se presentan con mayor frecuencia en la segunda porción con más de $4 \mathrm{~cm}$ de diámetro por lo que con frecuencia son susceptibles de ser sometidos a pancreatoduode-
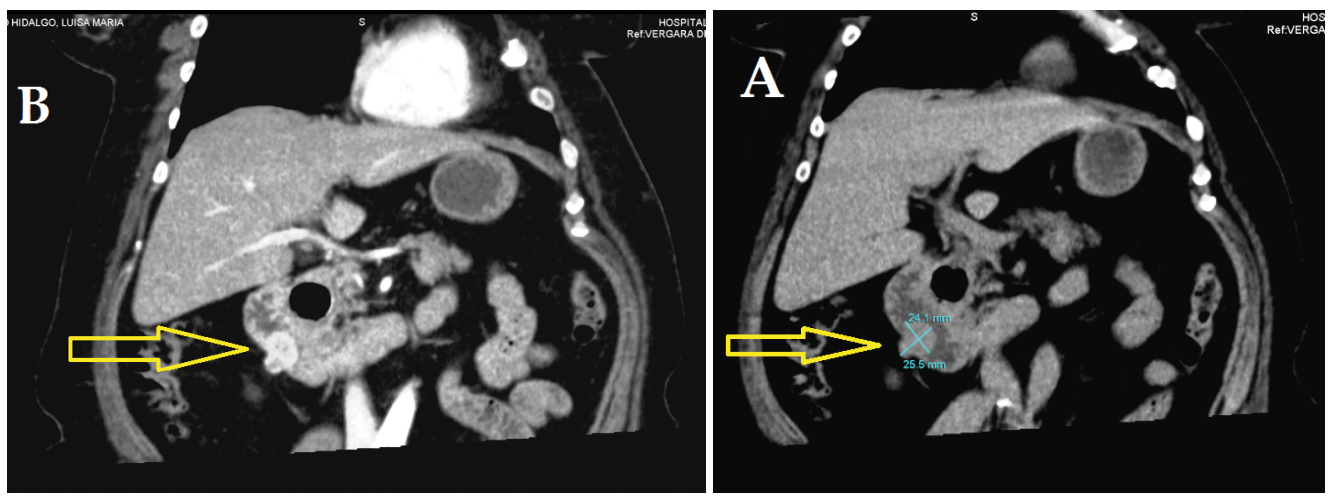

Figura 5. A. Vista coronal que muestra un GIST en la pared lateral de la tercera porción del duodeno que medía $3 \mathrm{~cm}$ de diámetro, en una paciente de 61 años de edad. B. El tumor se observaba bien definido y contrastado. La paciente se trató mediante resección en cuña la cual dejó un gran efecto en la pared duodenal, el que se reparó mediante duodenoyeyunostomía en Y de Roux. 

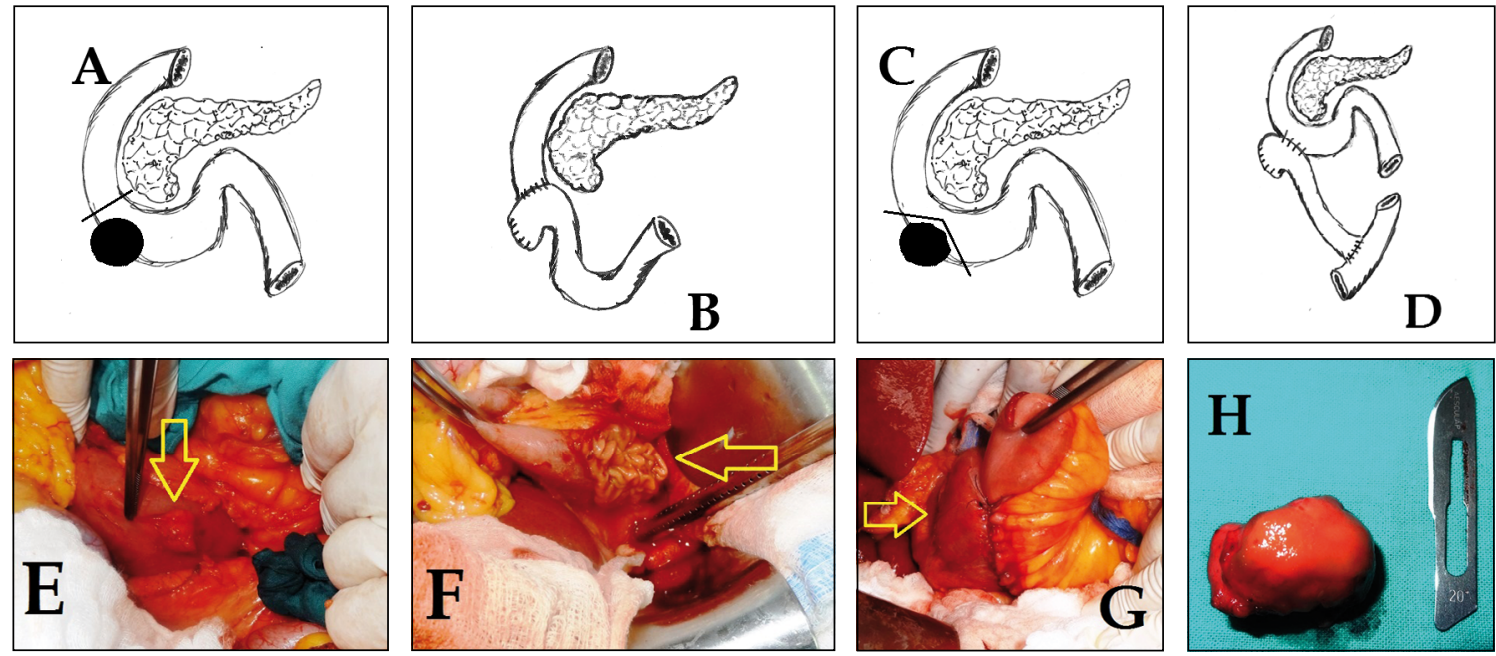

Figura 6. Procedimientos quirúrgicos para GIST de la tercera porción duodenal. A. Duodenectomía distal incluyendo la tercera y cuarta porción del duodeno. B. Reconstrucción de la duodenectomía distal mediante una duodeno-yeyunostomía termino-lateral. C. Resección en cuña de la tercera porción del duodeno. D. Reconstrucción de la resección mediante una duodeno-yeyuno anastomosis en Y de Roux. E. Crecimiento exofítico de GIST en la pared de la tercera porción duodenal. F. Defecto dejado en la pared duodenal por la resección en cuña del tumor. G. Reconstrucción mediante duodeno-yeyuno anastomosis en Y de Roux. H. Pieza quirúrgica.

nectomía ${ }^{19,20}$. La Tabla 5 resume las características, diferencias y similitudes entre pacientes operados mediante procedimientos locales comparados con tumores resueltos mediante pancreatoduodenectomía. Los pacientes sometidos a procedimientos

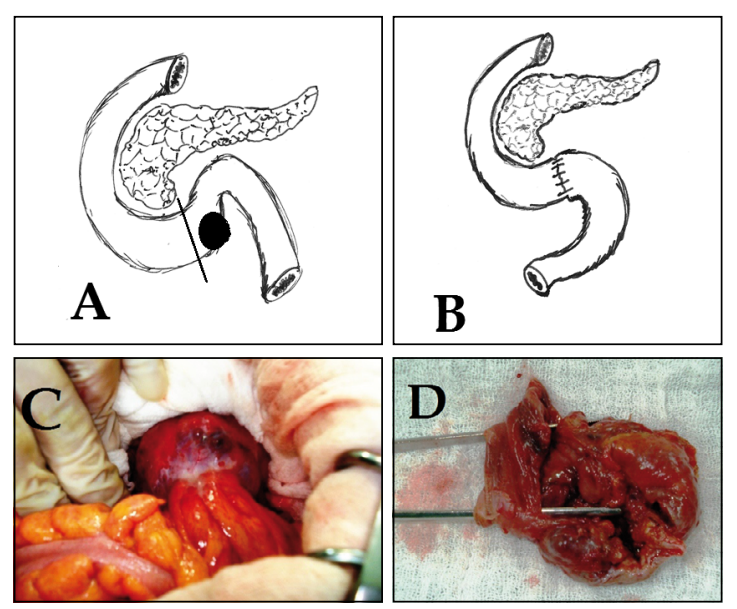

Figura 7. Procedimientos quirúrgicos para GIST de la cuarta porción duodenal. A. Duodenectomía distal incluyendo la cuarta porción del duodenal. B. Reconstrucción mediante duodeno-yeyunostomía primaria. C. GIST localizado en la cuarta porción del duodeno en una paciente de 38 años de edad operada de urgencia. D. Pieza quirúrgica mostrando la resección segmentaria de la cuarta porción del duodeno incluyendo el GIST. más complejos tienen un promedio de edad mayor a los sometidos a resecciones locales, se presentan con dolor abdominal, el tumor es mayor a $5 \mathrm{~cm}$ y se localiza en la segunda porción, los pacientes en los que se realizan resecciones locales con mayor frecuencia son habitualmente del género femenino, se presentan con hemorragia y el tumor es menor a $5 \mathrm{~cm}$. Debido a que no se diseminan por vía linfática no se realiza linfadenectomía, algunos autores que han realizado linfadenectomía en operaciones por GIST han encontrado metástasis en menos de $2 \%$ de todos los $\operatorname{casos}^{19}$. La completa resección del tumor en algunos casos requiere la resección de otros órganos y estructuras adyacentes ${ }^{44,45}$. La necesidad de efectuar procedimientos asociados es similar en pacientes sometidos a resecciones locales y pacientes sometidos a pancreatoduodenectomía ${ }^{19,44}$.

\section{Terapia adyuvante}

El mesilato de imatinib es la primera línea de tratamiento en pacientes con GIST avanzado, mejorando la sobrevivencia libre de enfermedad y la sobrevivencia general ${ }^{4,20,39}$. El imatinib ha sido aprobado para su uso en pacientes después de la resección de GIST con elevado riesgo de recurrencia a una dosis de $400 \mathrm{mg}$ diarios $^{39,57}$. El maleato de sunitinib es la segunda línea de tratamiento para pacientes con GIST refractario al imatinib ${ }^{10}$. 
Tabla 5. Características de GIST tratados mediante pancreatoduodenectomía o resección local ${ }^{7,19,20,44}$

\begin{tabular}{|lcc|}
\hline Variable & Resección local: cuña o segmentaria & Whipple \\
\hline Edad promedio (años) & $54-61$ & $56-65$ \\
Género femenino (\%) & $44-73$ & $17-50$ \\
Hemorragia (\%) & $24-68$ & $16-54$ \\
Dolor abdominal (\%) & $9-19$ & $4-37$ \\
Obstrucción intestinal (\%) & 3 & 3 \\
Masa abdominal (\%) & 9 & $4-17$ \\
Tamaño promedio del tumor (cm) & $3-5$ & $6-10$ \\
Primera porción duodenal (\%) & $9-14$ & $1-8$ \\
Segunda porción duodenal (\%) & $17-45$ & $37-83$ \\
Tercera porción duodenal (\%) & $27-64$ & $10-11$ \\
Cuarta porción duodenal (\%) & $18-29$ & $5-17$ \\
Procedimientos asociados (\%) & $8-31$ & $4-24$ \\
\hline
\end{tabular}

\section{Resistencia al imatinib}

La resistencia inicial al imatinib se presenta en $9 \%$ a $13 \%$ de todos los $\operatorname{casos}^{57}$ y puede ser primaria o secundaria de acuerdo con el período de tiempo entre el inicio del tratamiento y el desarrollo de metástasis o recurrencia local ${ }^{57-60}$. La resistencia precoz o primaria se presenta dentro de los primeros tres meses de tratamiento en $9 \%$ a $15 \%$ de todos los pacientes. La resistencia tardía o secundaria se desarrolla después de 24 meses de tratamiento en $44 \%$ de todos los pacientes ${ }^{58-60}$. Se han identificado factores de riesgo pronósticos para la predicción de la respuesta al imatinib, uno de los más importantes es el estado de mutación del KIT/PDGFRa; pacientes con mutaciones de los exones $11,13 \mathrm{y}$ 17 presentan una mejor respuesta ${ }^{58,60}$. Otros genes que predicen una buena respuesta se localizan en el cromosoma $19 \mathrm{p}^{58}$. Aproximadamente $85 \%$ a $90 \%$ de todos los GIST presentan mutaciones primarias del KIT y del PDGFR $\alpha^{58,59}$, en pacientes con resistencia primaria o secundaria se han identificado mutaciones secundarias de los exones $9,11,14$ y $17 \mathrm{del} c-k i t^{58}$. Mutaciones de los exones 11 y 13 se relacionan a resistencia primaria y mutaciones de los exones 11 y 17 a resistencia secundaria ${ }^{58,60,61}$. Pacientes con GIST metastásico o recurrente que inicialmente responden al imatinib y desarrollan resistencia deben ser explorados quirúrgicamente precozmente debido a que en ese momento las oportunidades de resección completa son mayores ${ }^{58-64}$. El uso de dosis mayores de imatinib después de la segunda cirugía incrementa la posibilidad de sobrevivencia libre de enfermedad por 24 meses o más, sin imatinib la sobrevivencia excepcionalmente es mayor a 12 meses $^{61,62}$. Una segunda generación de inhibidores de tirosina kinasa está constituida por el sunitinib que ha demostrado su utilidad en GIST resistentes a imatinib, particularmente en aquellos tumores con mutaciones de los exones 9, 13 y 14 asociados a resistencia primaria ${ }^{65,66}$. Eventualmente el sunitinib también desarrolla resistencia ${ }^{67,68}$ y en estos pacientes sólo un abordaje quirúrgico agresivo podrá brindar alguna oportunidad de superar la enferme$\mathrm{dad}^{67,69}$. Otro fármaco recientemente desarrollado con actividad en GIST resistentes al imatinib es el nilotinib que también tiene actividad en tumores resistentes al sunitinib ${ }^{70,71}$.

\section{Pronóstico}

La resección segmentaria o en cuña de GIST duodenales ha demostrado ser suficiente y curativa con una sobrevivencia libre de enfermedad satisfactoria en series compuestas específicamente por tumores del duodeno $0^{42-46}$. La Tabla pronóstica para GIST desarrollada el año 2001 por el taller de consenso patrocinado por National Institutes of Health y National Cancer Institute recientemente modificada por Joensuu ${ }^{42-46}$, es la utilizada para el pronóstico de estos pacientes (Tabla 4). La sobrevivencia a 5 años para tumores mayores a $10 \mathrm{~cm}$ es de $20 \%$ y la sobrevivencia en tumores menores a $5 \mathrm{~cm}$ llega a $65 \% 0^{9,19,42}$. Específicamente, en pacientes operados por GIST duodenal localizado la sobrevivencia global es de $98 \%$ a 3 años y $89 \%$ a 5 años, con una sobrevivencia libre de enfermedad de $67 \%$ a 3 años y $64 \%$ a 5 años $^{20}$. Todos los GIST se asocian 
a riesgo de recurrencia, $40 \%$ a $50 \%$ de todos los pacientes con resecciones potencialmente curativas desarrollan metástasis o recurrencias ${ }^{38}$. A pesar del conocimiento actual sobre la biología de los GIST, estos tumores se comportan erráticamente, aun en GIST de bajo riesgo se han reportado recurrencias 20 o más años después de la resección quirúrgica9 . En pacientes con GIST duodenal se han descrito recurrencias hasta en $39 \%$ de los $\operatorname{casos}^{21}$. En pacientes con tumores de alto riesgo, la recurrencia es inevitable. En pacientes con un seguimiento de 2 años, se han encontrado recurrencias en $19 \%$ con metástasis a distancia en $13 \%$ a $23 \%$, recurrencia local en $2 \%$ a $15 \%$ y recurrencia sincrónica local y a distancia en $4 \%{ }^{19,21}$. El lugar más común para el desarrollo de metástasis es el hígado ${ }^{19,21,42}$. Después de más de una década de investigación intensiva, las conclusiones son las mismas que hacen 10 años atrás, los factores determinantes de sobrevivencia más importantes son el recuento de mitosis y el tamaño tumoral ${ }^{7,19,20,21,42,43,45}$.

\section{Conclusiones}

La mayoría de los artículos sobre GIST duodenales incluyen en su introducción y discusión una frase indicando que el tratamiento quirúrgico óptimo para GIST duodenal no se encuentra definido ${ }^{17-21}$. Esto se debe a que no se han realizado estudios clínicos aleatorios y a que los reportes publicados constituyen la experiencia de uno o más centros de referencia. La revisión cuidadosa de esta literatura demuestra que los abordajes quirúrgicos para GIST duodenal son bastante estandarizados entre los diferentes autores. Todos toman en cuenta la localización del GIST en el duodeno y sus relaciones anatómicas para decidir el tipo de abordaje quirúrgico. Utilizando el sentido común de este conocimiento se proponen en este artículo, opciones quirúrgicas definidas para GIST duodenales.

\section{Referencias}

1. Joensuu H. Gastrointestinal stromal tumor (GIST). Ann Oncol. 200617 (Suppl. 10):280-6.

2. Mazur MT, Clark HB. Gastric stromal tumors: Reappraisal of histogenesis. Am J Surg Pathol. 1983;7:50719.

3. Hirota S, Isozaki K, Moriyama Y, Hashimoto K, Nishida $\mathrm{T}$, Ishiguro $\mathrm{S}$, et al. Gain-of-function mutations of $c$-kit in human gastrointestinal stromal tumors. Science 1998;279:577-80.

4. Joensuu H, Roberts P, Sarlomo-Rikala M, Andersson LC, Tervahartiala P, Tuveson D, et al. Effect of the tyrosine kinase inhibitor STI571 in a patient with metastatic gastrointestinal stromal tumor. N Engl J Med. 2001;344:1052-6.

5. Blay JY, Bonvalot S, Casali P, Choi H, Debiec-Richter M, Dei Tos AP, et al. Consensus meeting for the management of gastrointestinal stromal tumors: Report of the GIST Consensus Conference of 20-21 March 2004, under the auspices of ESMO. Ann Oncol. 2005; 16:56678.

6. Van den Abbeele AD, The lessons of GIST-PET and PET/CT: A new paradigm for imaging. Oncologist 2008;13(Suppl. 2):8-13.

7. Yang F, Jin C, Du Z, Subedi S, Jiang Y, Li J, et al. Duodenal gastrointestinal stromal tumor: Clinicopathological characteristics, surgical outcomes, long term survival and predictors for adverse outcomes. Am J Surg. 2013;206:360-7.

8. Agaimy A, Wünsch PH. Gastrointestinal stromal tumors: A regular origin in the muscularis propria, but an extremely diverse gross presentation. Langenbecks Arch Surg. 2006;391:322-9.

9. Corless CL, Fletcher JA, Heinrich MC. Biology of gastrointestinal stromal tumors. J Clin Oncol. 2004;22:3813-25.

10. Kitamura Y. Gastrointestinal stromal tumors: Past, present, and future. J Gastroenterol. 2008;43:499-508.

11. Bórquez PM, Neveu RC. Tumores del estroma gastrointestinal (GIST), un particular tipo de neoplasia. Rev Med Chile. 2008;136:921-9.

12. Vij M, Agrawal V, Kumar A, Pandey R. Cytomorphology of gastrointestinal stromal tumors and extra-gastrointestinal stromal tumors: A comprehensive morphologic study. J Cytol. 2013;30:8-12.

13. Beltrán MA, Vicencio AO, Barra MM, Contreras MA, Wilson CS, Cruces KS. Resultados del tratamiento quirúrgico de los tumores del estroma gastrointestinal (GIST) en la IV Región de Chile. Rev Chil Cir. 2011;63:290-6.

14. Beltrán MA, Pujado B, Méndez PE, Gonzáles FJ, Margulis DI, Contreras MA, et al. Gastric Gastrointestinal Stromal Tumor (GIST) incidentally found and resected during laparoscopic sleeve gastrectomy. Obes Surg. 2010;20:393-6.

15. Uchida H, Sasaki A, Iwaki K, Tominaga M, Yada K, Iwashita $\mathrm{Y}$, et al. An extramural gastrointestinal stromal tumor of the duodenum mimicking a pancreatic head tumor. J Hepatobiliary Pancreat Surg. 2005;12:324-7.

16. Huang CC, Yang CY, Lai IR, Chen CN, Lee PH, Lin MT. Gastrointestinal stromal tumor of the small intestine: A clinicopathologic study of 70 cases in the postimatinib era. World J Surg. 2009;33:828-34.

17. Chung JC, Chu CW, Cho GS, Shin EJ, Lim CW, Kim $\mathrm{HC}$, et al. Management and outcome of gastrointestinal stromal tumors of the duodenum. J Gastrointest Surg. 2010;14:880-3.

18. Lin C, Chang Y, Zhang Y, Zuo Y, Ren S. Small duode- 
nal gastrointestinal stromal tumor presenting with acute bleeding misdiagnosed as hemobilia: Two case reports. Oncology Letters 2012;4:1069-71.

19. Johnston FM, Kneuertz PJ, Cameron JL, Sanford D, Fisher S, Turley R, et al. Presentation and management of gastrointestinal stromal tumors of the duodenum: A multi-institutional analysis. Ann Surg Oncol. 2012;19:335160 .

20. Colombo C, Ronellenfitsch U, Yuxin Z, Rutkowski P, Miceli R, Bylina E, et al. Clinical, pathological and surgical characteristics of duodenal gastrointestinal stromal tumor and their influence on survival: A multi-center study. Ann Surg Oncol. 2012;19:3361-7.

21. Beham A, Schaefer IM, Cameron S, von Hammerstein K, Füzesi L, Ramadori G, et al. Duodenal GIST: A single center experience. Int J Colorectal Dis. 2013;28:581-90.

22. Fletcher CD, Berman JJ, Corless C, Gorstein F, Lasota J, Longley BJ, et al. Diagnosis of gastrointestinal stromal tumors: A consensus approach. Hum Pathol. 2002;33:459-65.

23. Rutkowski P, Bylina E, Wozniak A, Nowecki ZI, Osuch $\mathrm{C}$, Matlok M, et al. Validation of the Joensuu risk criteria for primary resectable gastrointestinal stromal tumor - The impact of tumor rupture on patient outcomes. European J Surg Oncol. 2011;37:890-6.

24. Beltrán MA, Barría C, Contreras MA, Wilson CS, Cruces KS. Tumor del estroma gastrointestinal (GIST) en una paciente con neurofibromatosis tipo 1. Rev Med Chile 2009;137:1197-200.

25. Relles D, Baek J, Witkiewicz A, Yeo CJ. Periampullary and duodenal neoplasms in neurofibromatosis type 1: Two cases and an updated 20-year review of the literature yielding 76 cases. J Gastrointest Surg. 2010;14:105261.

26. Agaimy A, Vassos N, Croner RS. Gastrointestinal manifestations of neurofibromatosis type 1 (Recklinghausen's disease): Clinicopathological spectrum with pathogenetic considerations. Int J Clin Exp Pathol. 2012;5:852-62.

27. Beltrán MA, Cruces KS, Barría C, Verdugo G. Multiple gastrointestinal stromal tumors of the ileum and neurofibromatosis type 1. J Gastrointest Surg. 2006;10:297-301.

28. Lee CM, Chen HC, Leung TK, Chen YY. Gastrointestinal stromal tumor: Computed tomographic features. World J Gastroenterology. 2004;10:2417-8.

29. Burkill GJ, Badran M, Al-Muderis O, Thomas JM, Judson IR, Fisher C, et al. Malignant gastrointestinal stromal tumor: Distribution, imaging features, and pattern of metastatic spread. Radiology 2003;226:527-32.

30. Hersh MR, Choi J, Garrett C, Clark R. Imaging gastrointestinal stromal tumors. Cancer Control 2005;12:111-5.

31. Kim JY, Lee JM, Kim KW, Park HS, Choi JY, Kim $\mathrm{SH}$, Kim MA, et al. Ectopic pancreas: CT findings with emphasis on differentiation from small gastrointestinal stromal tumor and leiomyoma. Radiology 2009;252:92100.

32. Lupescu IG, Grasu M, Boros M, Gheorghe C, Ionescu
M, Popescu I, et al. Gastrointestinal stromal tumors: Retrospective analysis of the computer-tomographic aspects. J Gastrointest Liver Dis. 2007;16:147-51.

33. Chourmouzi D, Sinakos E, Papalavrentios L, Akriviadis E, Drevelegas A. Gastrointestinal stromal tumors: A pictorial review. J Gastrointest Liver Dis. 2009; 18:379-83.

34. Buckley JA, Fishman EK. CT evaluation of small bowel neoplasms: Spectrum of disease. RadioGraphics 1998;18:379-92.

35. Ulusan S, Koc Z, Kayaselcuk F. Gastrointestinal stromal tumours: CT findings. Br J Radiol. 2008;81:51823.

36. Krause DS, van Etten RA. Tyrosine kinases as targets for cancer therapy. N Engl J Med. 2005;353:172-87.

37. Demetri GD, von Mehren M, Blanke CD, van den Abbeele AD, Eisenberg B, Roberts PJ, et al. Efficacy and safety of imatinib mesilate in advanced gastrointestinal stromal tumors. N Engl J Med. 2002;347:47280.

38. Ludvigsen L, Toxvaerd A, Mahdi B, Krarup-Hansen A, Bergenfeldt M. Succesful resection of an advanced duodenal gastrointestinal stromal tumor after downstaging with imatinib: Report of a case. Surg Today 2007;37:1105-9.

39. Rutkowski P, Gronchi A, Hohenberger P, Bonvalot S, Schöffski P, Bauer S, et al. Neoadjuvant imatinib in locally advanced gastrointestinal stromal tumors (GIST): The EORTC STBSG experience. Ann Surg Oncol. 2013;20:2937-43.

40. NCCN Clinical practice guidelines in oncology. Soft tissue sarcoma 2012, Version 2.2012.

41. The ESMO/European Sarcoma Network Working Group. Gastrointestinal stromal tumors: ESMO clinical practice guidelines for diagnosis, treatment and followup. Ann Oncol. 2012;23 (Suppl. 7):vii 49-55.

42. Wu TJ, Lee LY, Yeh CN, Wu PY, Chao TC, Hwang TL, et al. Surgical treatment and prognostic analysis for gastrointestinal stromal tumors (GISTs) of the small intestine: Before the era of imatinib mesylate. BMC Gastroenterology 2006;6:29.

43. Buchs NC, Bucher P, Gervaz P, Ostermann S, Pugin F, Morel P. Segmental duodenectomy for gastrointestinal stromal tumor of the duodenum. World J Gastroenterology 2010;16:2788-92.

44. Tien YW, Lee CY, Huang CC, Hu RH, Lee PH. Surgery for gastrointestinal stromal tumors of the duodenum. Ann Surg Oncol. 2010;17:109-14.

45. El-Gendi A, El-Gendi S, El-Gendi M. Feasibility and oncological outcomes of limited duodenal resection in patients with primary nonmetastatic duodenal GIST. J Gastrointest Surg. 2012;16:2197-202.

46. Bourgouin S, Hornez E, Guiramand J, Barbier L, Delpero JR, Le Treut YP, et al. Duodenal gastrointestinal stromal tumors (GISTs): Arguments for conservative surgery. J Gastrointest Surg. 2013;17:482-7. 
47. Yildirgan MI, Başoğ M, Atamanalp SS, Albayrak Y, Gürsan N, Önbaş Ö. Duodenal stromal tumor: Report of a case. Surg Today 2007;37:426-9.

48. Sakakima Y, Inoue S, Fujii T, Hatsuno T, Takeda $\mathrm{S}$, Kaneko $\mathrm{T}$, et al. Emergency pylorus-preserving pancreatoduodenectomy followed by second-stage pancreatojejunostomy for a gastrointestinal stromal tumor of the duodenum with intramural gas figure: Report of a case. Surg Today 2004;34:701-5.

49. Asakama M, Sakamoto Y, Kajiwara T, Nara S, Esaki M, et al. Simple segmental resection of the second portion of the duodenum for the treatment of gastrointestinal stromal tumors. Langenbecks Arch Surg. 2008;393:6059.

50. Morcos B, Al-Ahmad F. A large gastrointestinal stromal tumor of the duodenum: Report of a case. J Med Case Reports 2011;5:457.

51. Sakamoto Y, Yamamoto J, Takahashi H, Kokudo N, Yamaguchi T, Muto T, et al. Segmental resection of the third portion of the duodenum for a gastrointestinal stromal tumor: A case report. Jpn J Clin Oncol. 2003;33:364-6.

52. Mennigen R, Wolters HH, Schulte B, Pelster FW. Segmental resection of the duodenum for gastrointestinal stromal tumor (GIST). World J Surg Oncol. 2008;6:105.

53. Ghidirim G, Mishin I, Gagauz I, Vozian M, Cernii A, Cernat M. Duodenal gastrointestinal stromal tumor. Rom J Morphol Embryol. 2011;52(3 Suppl):1121-5.

54. Shaw A, Jeffery J, Dias L, Nazir S. Duodenal wedge resection for large gastrointestinal stromal tumor (GIST) presenting with life-threatening hemorrhage. Case Reports Gastrointest Med. 2013;2013:562642.

55. Sasaki A, Koeda K, Obuchi T, Nakajima J, Nishizuka $\mathrm{S}$, Terashima M, et al. Tailored laparoscopic resection for suspected gastric gastrointestinal stromal tumor. Surgery 2010;147:516-20.

56. Chen YH, Lu KH, Yeh CN, Hsu JT, Liu YY, Tsai CY, et al. Laparoscopic resection of gastrointestinal tumors: Safe, efficient, and comparable oncologic outcomes. J Laparoendosc Adv Surg Tech. 2012;22:758-63.

57. Bickenbach K, Wilcox R, Veerapong J, Kindler HL, Posner MC, Noffsinger A, et al. A review of resistance patterns and phenotypic changes in gastrointestinal stromal tumors following imatinib mesylate therapy. J Gastrointest Surg. 2007;11:758-66.

58. Wang CM, Huang K, Zhou Y, Du CY, Ye YW, Fu H, et al. Molecular mechanisms of secondary imatinib resistance in patients with gastrointestinal tumors. J Cancer Res Clin Oncol. 2010;136:1065-71.

59. Rink L, Skorobogatko Y, Kossenkov AV, Belinsky MG, Pajak T, Heinrich MC, et al. Gene expression signatures and response to imatinib mesilate in gastrointestinal stromal tumor. Mol Cancer Ther. 2009;8:2172-82.

60. Gounder MM, Maki RG. Molecular basis for primary and secondary tyrosine kinase inhibitor resistance in gastrointestinal stromal tumor. Cancer Chemother Pharmacol. 2011;67:25-43.

61. Yeh CN, Chen TW, Tseng JH, Liu YY, Wang SY, Tsai $\mathrm{CY}$, et al. Surgical management in metastatic gastrointestinal stromal tumor (GIST) patients after imatinib mesilate treatment. J Surg Oncol. 2010;102:599-03.

62. Andtbacka RH, Ng CS, Scaife CL, Cormier JN, Hunt KK, Pisters PW, et al. Surgical resection of gastrointestinal stromal tumors after treatment with imatinib. Ann Surg Oncol. 2007;14:14-24.

63. D'Amato G, Steinert DM, McAuliffe JC, Trent JC. Update on the biology and therapy of gastrointestinal stromal tumors. Cancer Control 2005;12:44-55.

64. Jiang WZ, Guan GX, Lu HS, Yang YH, Kang DY, Huang HG. Adjuvant imatinib treatment after R0 resection for patients with high-risk gastrointestinal stromal tumor: A median follow-up of 44 months. J Surg Oncol. 2011;104:760-4.

65. Blay JY. Pharmacological treatment of gastrointestinal stromal tumors: An update on the role of sunitinib. Ann Oncol. 2010;21:208-15.

66. Younus J, Verma S, Franek J, Coakley N. Sunitinib maleate for gastrointestinal stromal tumor imatinib mesylate-resistant patients: Recommendations and evidence. Current Oncol. 2010;17:4-10.

67. Kikuchi H, Setoguchi T, Miyazaki S, Yamamoto M, Ohta M, Kamiya K, et al. Surgical intervention for imatinib and sunitinib-resistant gastrointestinal stromal tumors. Int J Clin Oncol. 2011;16:741-5.

68. Wang WL, Conley A, Reynoso D, Nolden L, Lazar AJ, George S, et al. Mechanisms of resistance to imatinib and sunitinib gastrointestinal stromal tumor. Cancer Chemother Pharmacol. 2011;67:15-24.

69. Dudeja V, Armstrong LH, Gupta P, Ansel H, Askari S, Al-Refaie WB. Emergence of imatinib resistance associated with downregulation of c-kit expression in recurrent gastrointestinal stromal tumor (GIST): Optimal timing of resection. J Gastrointest Surg. 2010;14:55761.

70. Montemurro M, Bauer S. Treatment of gastrointestinal stromal tumor after imatinib and sunitinib. Curr Opin Oncol. 2011;23:367-72.

71. Sawaki A, Nishida T, Doi T, Yamada Y, Komatsu Y, Kanda T, et al. Phase 2 study of nilotinib as third-line therapy for patients with gastrointestinal stromal tumor. Cancer 2011;117:4633-41. 